\title{
Intensive resistance exercise and circadian salivary testosterone concentrations among young male recreational lifters
}

\begin{abstract}
Strength and morphological adaptations to resistance exercise are mediated in part by anabolic hormones such as testosterone, yet the time course of variability in circadian hormone concentrations is not well characterized. This study, investigated how the circadian rhythm of salivary testosterone is altered by resistance exercise in young men. Twenty healthy young male recreational lifters (age, $18.0 \pm 1.3$ years) with 2 years of experience in weightlifting were recruited. A randomized controlled trial was conducted, and subjects were randomly assigned to either the resistance exercise group $(n=10)$, who completed a series of resistance exercise ( 3 times a week, in the afternoon, 6-7 repetitions, at 85\% of 1 repetition maximum for 3 weeks), or a control group $(n=10)$, who did not exercise during the 3 weeks. Before and after the study, an unstimulated saliva sample $(2 \mathrm{ml})$ was taken every 2 hours for a maximum of 16 hours during each day. A significant decrease was observed in the resistance exercise $(44.2 \%, \mathrm{p}=0.001)$ and control group $(46.1 \%, \mathrm{p}=0.001)$ for salivary testosterone at each time point compared with baseline $(\mathrm{p}=0.001)$. There was also no significant difference between the exercise and resting conditions in both groups for salivary testosterone $(\mathrm{p}>0.05)$, except a significantly higher increase by $38.4 \%$ vs. $-0.02 \%$ ( $\mathrm{p}=$ 0.001), at 1730 hours during exercise sessions in the resistance exercise group compared with the control group. Resistance exercise has no noteworthy effect on circadian secretion of salivary testosterone throughout the 16 waking hours. These results indicate that athletes can undertake resistance exercise in either the morning or afternoon with the knowledge that a similar testosterone response can be expected regardless of the time of day.
\end{abstract}

Keyword: Androgens; Chronobiology; Saliva samples 